\title{
Le drainage agricole après la réforme de la $\mathrm{PAC}^{*}$
}

\author{
Etienne Frejefond ${ }^{(1)}$, Daniel Zimmer ${ }^{(1)}$, Pierre Vaquié(2), Michel Lagoutte ${ }^{(3)}$ \\ (1) Cemagref, Division Ouvrages pour le drainage et l'Etanchéité, Antony \\ ${ }^{2}$ FNCUMA, Paris; ${ }^{3}$ Géomètre, Nangis
}

De 1980 à 1988 le rythme annuel des travaux de drainage agricole par tuyaux enterrés s'est maintenu en France à plus de 125000 ha par an. Ce rythme a conduit à la réalisation du drainage de plus d'un million d'hectares pendant la décennie 80. Les superficies drainées en France représentaient en 1988 lors du dernier recensement général de l'agriculture 2,5 millions d'hectares (SCEES, 1991) dont 2 millions par tuyaux enterrés. Ce dernier chiffre s'est accru aujourd'hui de plus de 300000 ha. Avec un taux de couverture des besoins de $30 \%$ environ, la France se situe derrière les autres pays du nord de l'Europe dont les besoins sont pour la plupart couverts à plus de $60 \%$. En termes de proportion des superficies agricoles utiles, la France a draine environ $8 \%$, les taux du nord de l'Europe se situant entre 10 et 90\% (Figure 1).

A la suite des années de sécheresse qui ont marqué la fin des années 80 et devant les incertitudes de la nouvelle politique agricole commune, les réalisations de drainage ont vu leur volume annuel chuter au niveau de 1974-1975, soit environ 40000 ha/an. Cet effondrement du marché a eu des répercussions très importantes sur l'ensemble de la filière et plus particulièrement chez les entrepreneurs et les bureaux d'études dont une grande majorité a dû se reconvertir ou se résigner à disparaître.

Où en est-on aujourd'hui ? Quels sont les éléments d'explication de cet effondrement? Et quel est l'avenir du drainage dans le nouveau contexte de I'après-PAC? Avec l'appui du SNED' et du ministère de l'Agriculture et de la Pêche, un groupe de travail de l'AFEID a collecté des informations sur la répartition et les tendances dans les différents régions et départements, ceci avec le double objectif de comprendre et de donner des pistes pour raisonner les investissements des entreprises de drainage, des collectivités territoriales et des maîtres d'ouvrages.

\section{I 固 LES FAITS}

De 115000 ha en 1990, le marché est passé à 36000 ha en 1993 (Tableau 1). La chute a été de $30 \%$ environ en 90 et 91 , elle est passée par un maximum de $34 \%$ en 1992 puis s'est ralentie à $6 \%$ en 1993. La chute globale s'établit donc à près de $70 \%$. Elle est fortement marquée dans le Bassin Parisien et les Pays de

* Cet article reprend pour une large part un article paru dans la revue Géonelre en juillet 1995.

'Syndicat national des entreprises de drainage er d'hydraulique.

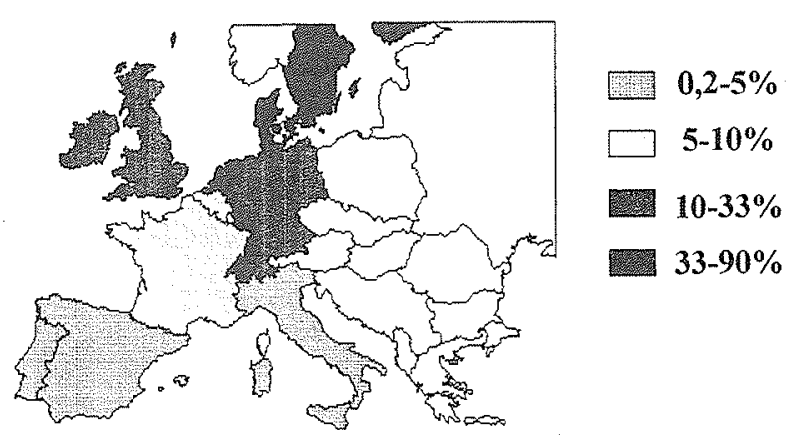

1. Part de la supercifie agricole utile drainée en Europe de l'Ouest.

Loire. Par contre a l'Est d'une ligne Bordeaux-Nancy, le drainage semble avoir relativement mieux résisté (Figure 2).

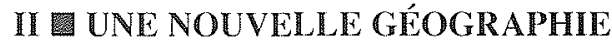 DU DRAINAGE ...}

Suite aux quotas laitiers en 1984, le drainage s'était fortement développé dans l'Ouest de la France èt en particulier dans la région Pays de Loire. De manière générale, les systèmes de polyculture élevage ont maintenu un rythme important de drainage pendant toute la seconde moitié des années 80 . Le drainage permettait à ces systèmes de se diversifier en introduisant ou en augmentant leur production en céréales.

A la veille de la crise, en 1988 et 1989, la géographie du drainage est encore marquée par cette tendance: une bonne part des superficies drainées se situent dans les Pays de Loire et dans la

\begin{tabular}{|l|c|c|c|c|c|}
\hline & 1989 & 1990 & 1991 & 1992 & 1993 \\
\hline $\begin{array}{l}\text { travaux } \\
\text { milliers } \\
\text { d'ha }\end{array}$ & 115 & 82 & 58 & 38,5 & 36 \\
\hline $\begin{array}{l}\text { réduc- } \\
\text { tion }\end{array}$ & & $-29 \%$ & $-30 \%$ & $-34 \%$ & $-6 \%$ \\
\hline
\end{tabular}

Tableau i. Evolution des superficies drainées par tuyaux enterrés en France de 1989 à 1993. 
frange sud du Bassin Parisien. La Bourgogne, la région MidjPyrénnées et le département du Nord constituent les autres bastions du drainage. De nombreux départements maintiennent un rythme supérieur à 3000 ha. La Loire Atlantique et la Vendée battent des records avec des superficies supérieures à 8000 ha.

Les années 90 et 91 ne modifient pas fondamentalement cette géographie du drainage. Il semble qu'en gros la répartition reste identique mais que les superficies drainées se réduisent un peu partout. Le rythme annuel le plus élevé reste celui de la Loire Atlantique avec 6200 ha.

A partir de 92 et 93, un changement plus profond s'opère. Aucun département ne se maintient au dessus des 2000 ha par an. L'importance des départements de l'Ouest et notamment des Pays de Loire a entièrement disparu. La nouvelle géographie du drainage fait apparaître une préminence des départements de ce que les débats sur l'aménagement du territoire ont nommé "la diagonale du vide " et qui s'étend de la Lorraine à MidiPyrénnées en passant par la Bourgogne et le Massif Central (Figure 2). De fait, un examen attentif montre que l'essentiel des départements situés sur cette diagonale ont vu leur rythme de drainage se maintenir peu ou prou voire, comme dans le Massif Central, augmenter légèrement. On note également un maintien relatif dans les départements des Landes et du Nord.

\section{III 湢 ... MAIS PEU DE MODIFICATIONS DE LA STRUCTURE DU MARCHÉ}

Si la géographie a changé, l'organisation du marché semble avoir évolué dans la droite ligne des années 80.

La maîtrise d'ouvrage se partage pour moitié entre maîtrise d'ouvrage collective (Associations Syndicales Autorisées, Associations Foncières...) et maîtrise d'ouvrage individuelle. Cette proportion moyenne est identique à celle qu'ont connue les années 80. Cette stabilité semble toutefois cacher des disparités ou de nouvelles tendances plus favorables au drainage individuel qui peuvent s'expliquer par la réduction, voire la suppression des subventions dans certains départements (Bretagne notamment) et la mise en application de la Loi sur l'Eau qui impose des déclarations à partir de superficies de 20 ha et des demandes d'autorisation au dessus de 100 ha.

De même, les parts respectives des entreprises et du secteur coopératif sont restées dans les proportions de ce qu'elles furent dans les années 80 . Une très lente érosion de la part des entreprises s'observe. Cette érosion avait démarré dès les années 80 , la part du secteur coopératif étant passée de $18 \%$ en 1980 à $29 \%$ en 1990. Depuis 1990, cette part a oscillé entre 29 et $31 \%$.

Concernant la taille des chantiers, peu d'éléments sont disponibles. La comparaison des données collectées au cours de l'en- quête révèle toutefois que la proportion des collecteurs dans le linéaire total s'est accrue, passant de $22 \%$ à $31 \%$ entre 1990 et $1993^{(2)}$. Cette tendance pourrait tout d'abord être due à une diminution de la taille des chantiers ; comme un minimum de collecteurs est dans tous les cas nécessaire, une réduction de taille devrait en effet se traduire par une augmentation de la proportion de ces derniers. Un autre élément d'explication peut venir d'une augmentation des réhabilitations de réseaux de drainage, suite à de grands travaux (TGV, autoroutes...). Ces réhabilitations requièrent en effet une part plus importante de drains de gros diamètre.

\section{IV 圆 A COMBIEN LE MARCHÉ DU DRAINAGE PEUT-IL ETRE ESTIMÉ AUJOURD'HUI ?}

Des éléments relatifs aux coûts du drainage sont recueillis chaque année par le bureau de l'Hydraulique Agricole et des Grands Ouvrages du ministère de l'Agriculture, de la Pêche et de l'Alimentation. Ces éléments sont relatifs aux superficies faisant l'objet de subventions; ils incluent des informations sur le drainage à la parcelle, sur les infrastructures collectives de drainage et sur les travaux d'assainissement superficiel par réseaux de fossés (Tableau 2).

La part des superficies drainées faisant l'objet de subventions se réduit légèrement de 1991 à 1993 , passant de $72 \%$ à $66 \%$ des superficies totales. Poursuivant une logique de cohérence des aménagements, les subventions sont ciblées prioritairement sur les infrastructures collectives de drainage enterré (taux moyen légèrement supérieur à $50 \%$ ), le taux de subvention du drainage à la parcelle étant d'environ $30 \%$. L'assainissement agricole bénéficie également de taux élevés, proches de $50 \%$.

Les coûts des travaux faisant l'objet de subventions montrent un coût du drainage à la parcelle en légère augmentation de 91 à 93 , passant de $8500 \mathrm{~F} /$ ha à près de $9500 \mathrm{~F} / \mathrm{ha}$. Ce coût passe à 12 ou $13000 \mathrm{~F} / \mathrm{ha}$ lorsqu' on lui ajoute les infrastructures collectives. La variabilité régionale des coûts est très grande : pour le drainage à la parcelle, l'échelle est en effet de 1 à 3 entre la Bretagne où le coût moyen est de l'ordre de $5000 \mathrm{~F} / \mathrm{ha}$ et des régions comme la Lorraine, le Limousin ou l'lle de France où il est de l'ordre de 14 à $15000 \mathrm{~F} / \mathrm{ha}$.

Rapportés aux superficies totales, ces chiffres fournissent une estimation du marché du drainage chutant de $638 \mathrm{MF}$ en 1991 à 433 MF en 1993. Avec l'assainissement agricole, le marché est de l'ordre de $535 \mathrm{MF}$ en 1993. Le montant des subventions attribuées représentait au total $158 \mathrm{MF}$ pour le drainage et l'assainissement agricole.

\footnotetext{
${ }^{21}$ Source : Syndicat national des fabricants de drain annele.
}

\begin{tabular}{|l|r|r|c|}
\hline Année & 1991 & 1992 & 1993 \\
\hline Superficies subventionnées (milliers d'ha) & 42 & 27 & 24 \\
\hline Coût moyen du drainage à la parcelle (F/ha) & 8580 & 9680 & 9370 \\
\hline Coût moyen du drainage et des infrastructures (F/ha) & 11010 & 13524 & 11964 \\
\hline Estimation du marché du drainage (MF/an) & 638 & 522 & 433 \\
\hline $\begin{array}{l}\text { Estimation du marché du drainage et de } \\
\text { l'assainissement agricole (MF/an) }\end{array}$ & 813 & 680 & 535 \\
\hline
\end{tabular}

Tableau 2. Estimation du marché du drainage de 1991 à 1993 (source Ministère de l'Agriculture, de la Pêche et de l'Alimentation). 


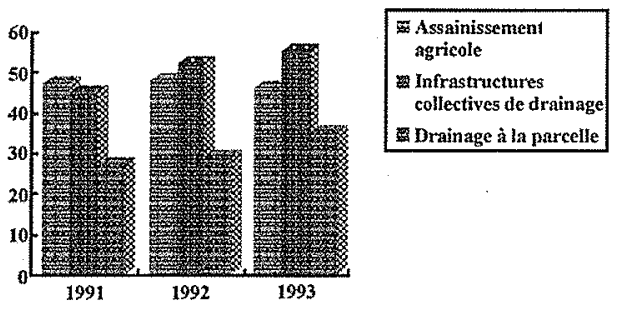

Figure 3. Evolution des taux moyens de subvention des travaux de drainage et d'assainissement agricole. (source Ministere de l'Agriculture et de la Pêche)

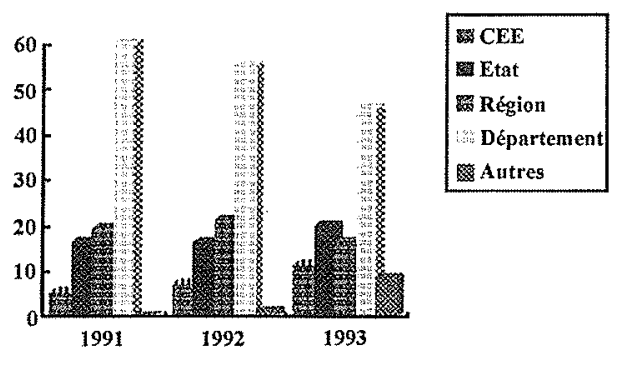

Figure 4. Part des differentes origines des subventions du drainage et de l'assainissement agricoles.

La part la plus importante des subventions est attribuée par les départements. Cette source est cependant en régression puisqu'elle est passće de $60 \%$ à $45 \%$ du total attribué entre 1991 et 1993. L'Etat et la Région constituent les seconds pourvoyeurs de subventions avec une contribution à peu près constante de $16 \%$ à $20 \%$ du total attribué. La CEE et les autres sources de subventions constituent le restant ; leur part est en augmentation entre 1991 et 1993 (Figures 3 et 4).

\section{V 圆 LES RAISONS DE L'EFFONDREMENT}

L'enquête ayant permis de recueillir les éléments statistiques ci-dessus a été réalisée en 1994. Elle a été l'occasion de recueillir l'avis des différents intervenants du drainage sur l'effondrement du marché. Deux facteurs retiennent majoritairement l'attention : le climat et la PAC.

Le climat de la fin des années 80 et du début des années 90 a été sec, ce qui a eu pour effet de reporter les investissements en hydraulique agricole du drainage vers l'irrigation dans plusieurs régions.

La Politique Agricole Commune a eu un effet important sur les investissements du monde agricole en général, effet surtout psychologique -et donc difficile à quantifier- au début des années 90 , les agriculteurs ne sachant pas encore quelles seraient les conséquences précises de la réforme sur leurs trésoreries et sur leurs revenus. Le climat d'incertitude qui régnait alors, a conduit à de nombreux reports d'investissements.

Trois autres types de facteurs sont assez souvent évoqués : les subventions accordées aux programmes de drainage, le taux d'équipement en drainage, élevé dans certains départements, notamment céréaliers et enfin les pressions en faveur d'une plus grande protection de l'environnement (Bretagne tout particulièrement).
Pour l'ensemble des personnes ayant répondu, la cause avancée n'est pas unique. C'est plutôt la conjonction d'un ensemble de facteurs défavorables qui est avancée.

A partir de ces appréciations, plusieurs facteurs ont été analysés plus précisément :

1. le climat;

2. la réforme de la PAC;

3. le taux et les besoins en drainage

4. les coûts et le marché du foncier ;

5. les subventions.

De ces différents facteurs, seuls les deux premiers, le climat et la PAC, ont pu être mis en relation avec l'évolution du marché, confirmant par là même les appréciations qualitatives recueillies lors de l'enquête. La PAC permet de comprendre les évolutions à l'échelle nationale alors que le climat semble expliquer une modulation des évolutions à l'échelle régionale. Le rôle des autres facteurs n'a pu être mis en évidence à ces échelles nationale et régionale, ce qui ne veut pas dire qu'ils n'ont pas leur importance à d'autres niveaux comme ceux du département ou de la petite région agricole.

\subsection{L'influence de la PAC}

La réforme de Ia PAC a été décidée en mai 1992. Son principal objectif est de parvenir à une maitrise des productions et notamment des productions de céréales et d'oléo-protéagineux. Les moyens choisis pour y parvenir, tels la jachère, doivent conduire à une extensification de la production, tout au moins à l'échelle nationale.

Depuis le début des années 90 les agriculteurs et toute la filière agricole ont vécu dans l'incertitude, attendant de mieux connaitre les effets de la réforme. Cette incertitude a eu pour effet de retarder de nombreux investissements, et plus particulièrement ceux qui, comme le drainage, sont associés à une intensification de la production. Au début des années 90, les effets sont donc surtout psychologiques et difficiles à appréhender. Ils sont par ailleurs diversifiés en fonction des systèmes de production : schématiquement, les systèmes céréaliers doivent être les plus touchés, les systèmes extensifs à base de bovins viande les plus favorisés.

Pour expliquer les évolutions du drainage nous avons donc recherché les correspondances possibles avec les conséquences prévues de la nouvelle PAC. Les évolutions anticipées des Revenus Bruts d'Exploitation (RBE) estimées par rapport à la référence de 1991, en supposant que les systèmes de production ne changent pas [1] nous ont servi de base.

A l'exception de l'Ouest de la France, et de quelques départements isolés, les prévisions d'évolution du RBE et du drainage se recoupent d'une manière tout-à-fait remarquable (Figure 5). Les départements à forte diminution du drainage correspondent aux départements à forte diminution prévisible du RBE (chute de plus de $4 \%$ ) ; elles correspondent aussi pour la plupart aux départements abritant des exploitations céréalières ou de grande culture [2]. Les départements où le drainage se maintient sont ceux où le RBE doit peu chuter ou s'améliorer.

Dans l'Ouest, et notamment dans les régions Bretagne et Pays de Loire la correspondance est moins bonne. Les prévisions d'évolution du RBE sont plutôt à la hausse alors que les chutes sont fortes. Une explication possible tient au fait que les main- 


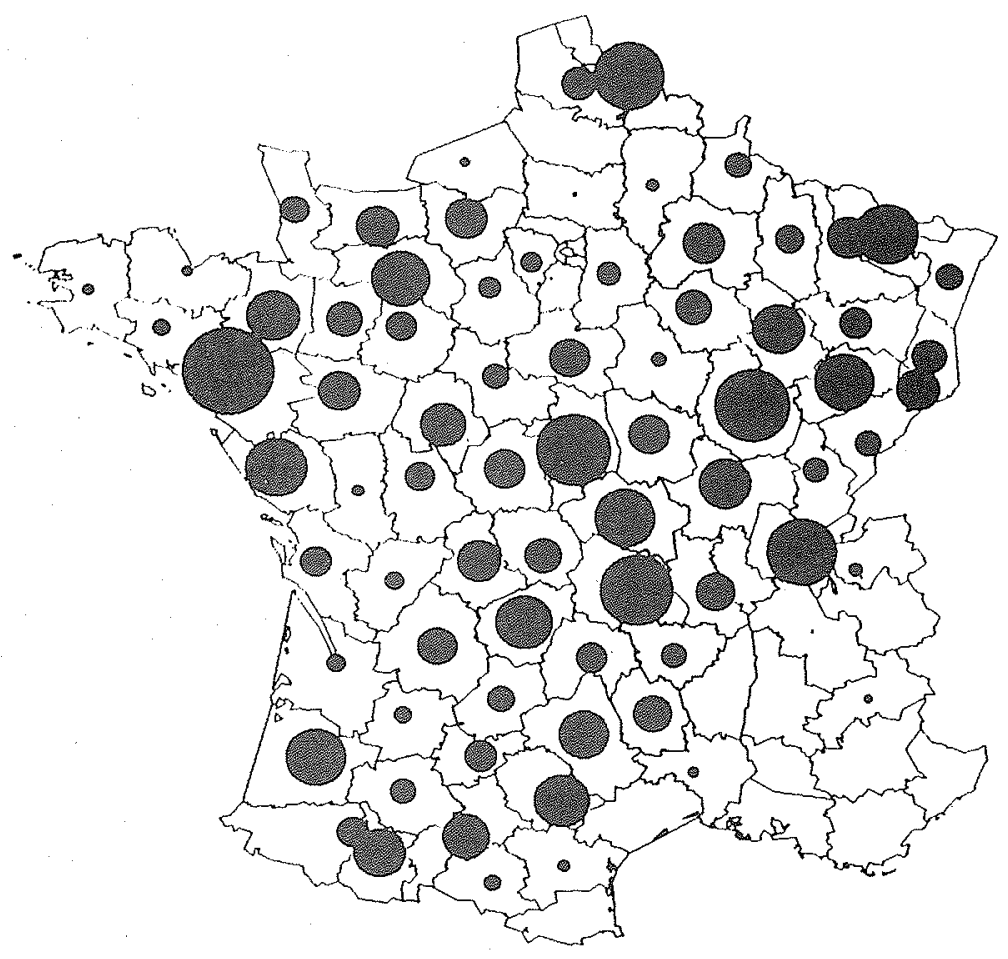

\section{Superficies en ha}
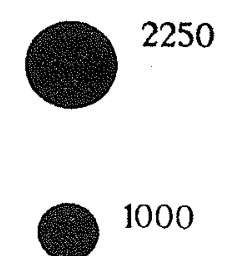

200

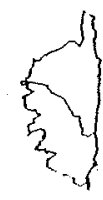

2. Superficies drainées en France par département en 1993.

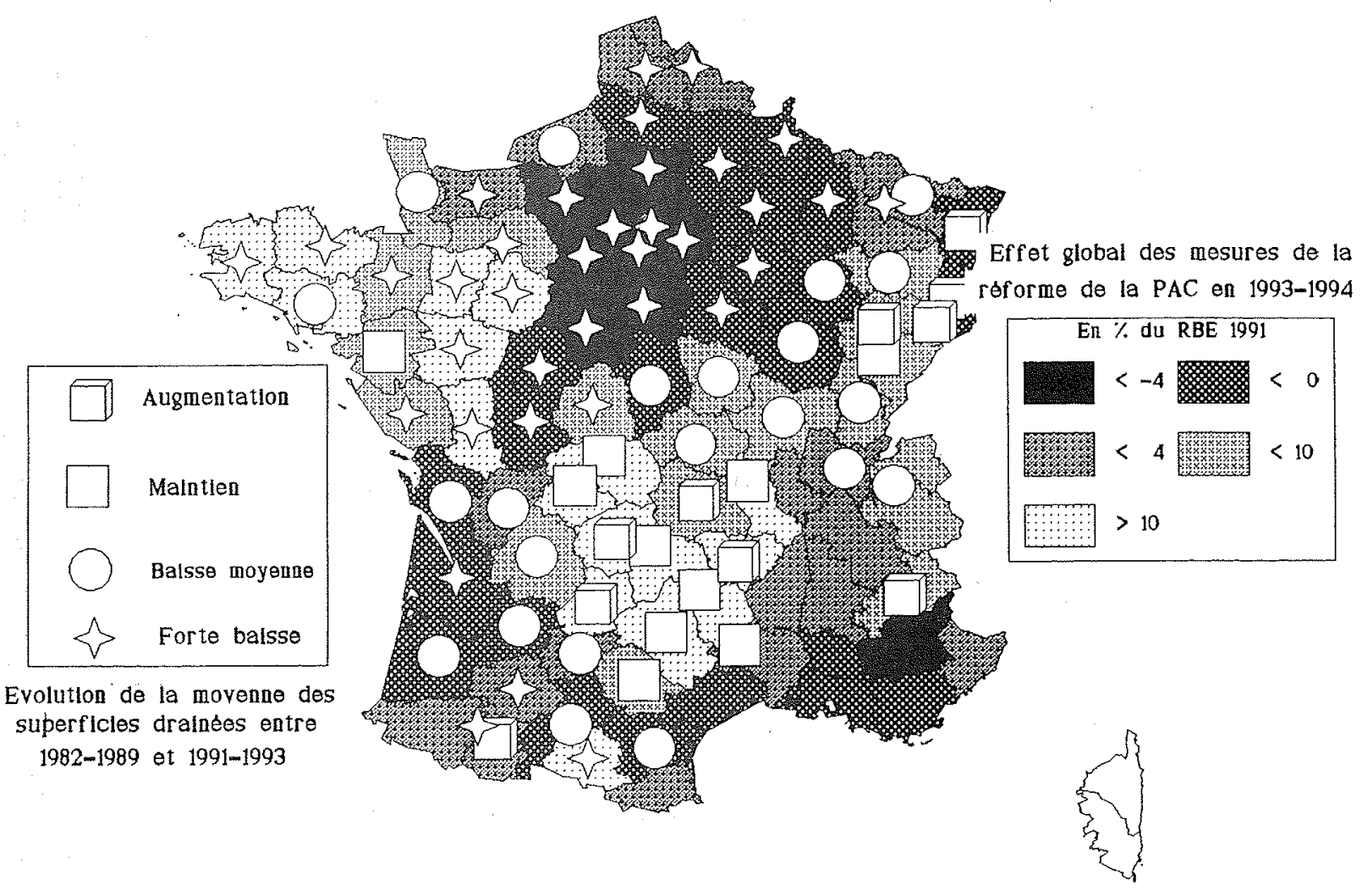

5. Impact de la réforme de la PAC sur les investissements en drainage entre 1991 et 1993. Sources : Effet de la PAC sur les revenus - Compte de l'agriculture 1991.

Evolution des superficies drainées 1990 et enquête 1991-1993. 
tiens ou augmentations de RBE prévus s'expliquent par la baisse du coût des céréales pour les nombreux élevages hors-sols de la région. Ces augmentations peuvent donc s'accompagner d'une perte d'intérêt pour des cultures qui étaient l'une des principales raisons d'être du drainage. Il est quasi certain par ailleurs que la sensibilité à l'environnement a également joué notamment en Bretagne, touchée par les pollutions par les nitrates.

\subsection{L'influence du climat}

L'idée que le drainage se décide à la suite de difficultés particulières d'entrée sur les parcelles est répandue dans la profession des draineurs. Dès lors, il n'est pas très surprenant qu'au contraire les investissements se réduisent en périodes sèches. Mais quelle peut-être l'ampleur du phénomène et peut-on le détecter?

Lés bulletins climatiques mensuels de la Météorologie Nationale 151 ont été utilisés pour déterminer, région par région, l'importance possible des contraintes d'excès d'eau depuis l'hiver 86-87 jusqu'à 92-93. Des indices globaux d'excès d'eau ont été déterminés en tenant compte à la fois des pluies d'automne et des pluies de printemps. Les évolutions régionales des superficies drainées ont été ensuite mises en correspondance avec ces indices.

Les résultats confirment que la pluviométrie ou du moins les écarts de pluviométrie d'une année à l'autre sont souvent explicatifs des évolutions des réalisations du drainage. C'est principalement dans les régions où la tendance n'est pas unique que les modulations par la pluviométrie sont nettes (ex. MidiPyrénées, Bourgogne, Alsace). Lorsque les chutes sont très fortes, les correspondances apparaissent moins (ex. la Lorraine, Centre Champagne).

Un autre résultat marquant : les superficies drainées sont mieux corrélées aux indices pluviométriques du printemps de l'année et de l'automne précédent qu'aux indices de l'automne et du printemps de l'année précédente. Le drainage, investissement de long terme, semble bien se décider dans le court terme!

\section{Q QUELQUES CONCLUSIONS}

1. La chute du marché du drainage a fortement désorganisé la filière : disparition de plus des deux tiers des entreprises, disparition d'un savoir-faire important. Certaines entreprises subsistent, généralement parce qu'elles ont réussi à se diversifier, notamment dans le génie civil (pose de câbles, drainage routier, création de retenues). La forte compétitivité qui caractérisait le marché du drainage agricole les a vraisemblablement aidées dans leur diversification. Cette tendance pourrait avoir un effet inflationiste sur les coûts du drainage.

Cette chute accompagne un doute de la profession agricole sur son avenir, sur le sens même à donner à son développement dans les conditions technico-économiques actuelles. Cette difficulté à retrouver un sens à des décisions techniques est alimentée par la logique de la nouvelle PAC et par la volonté de notre société d'une préservation accrue de notre environnement. Même si la logique qui prévaut à l'échelle de l'exploitation agricole peut être disjointe de la logique macro-économique, une cohérence minimale est nécessaire dans la prise de décision. D'une certaine façon la crise actuelle souligne la place d'investissement à long terme, structurant pour le développement des exploitations agricoles, du drainage.

Paradoxalement la déstructuration du marché du drainage présente des risques pour la cohérence des aménagements au sein des bassins versants. En effet la tendance est à un morcellement plus grand des chantiers et à une régression de la technicité et de la nécessaire réflexion qui devrait accompagner ce type d'investissement. Ces risques sont encore accrus par les imprécisions de la Loi sur l'Eau en matière de drainage [6].

2. La chute du marché semble s'être arrêtée depuis 1993. Les chiffres du SNFDA des linéaires de drains vendus en 1994 sont en augmentation de $20 \%$ par rapport à 1993 . Le marché du drainage et de l'assainissement agricoles représente aujourd'hui encore un marché de plus de $500 \mathrm{MF}$ par an, ce qui en fait le plus important de l'Union Européenne. Dans les conditions actuelles, ce marché témoigne que les besoins restent importants.

3. Dans ces nouvelles conditions quelle politique de subventionnement mettre en place ? Et quelles solutions techniques envisager ? Certaines régions et départements ont réduit leur soutien à l'assainissement agricole. D'autres, comme la profession agricole, sont dans l'incertitude, comme le sont actuellement les techniciens ayant à conseiller les agriculteurs en tentant de concilier les multiples impératifs du développement local et de l'aménagement des bassins versants. De tout évidence, une réflexion s'impose.

\section{Bibliographie}

[1] DESRIERS M., PERREL B., STRAUSS J.P., 1994 - Réforme de la PAC: des aides plus favorables à l'élevage qu'aux grandes cultures - Vers une réduction des disparités geographiques de revenu. Economie Rurale, 220-221, 119-123

[2] NEVEU A., 1993 - Les Nouveaux territoires de l'agriculture française. Uni-éditions, $175 \mathrm{p}$.

[3] SCEES, 1991 - Irrigation et drainage en France. Revue Agreste, Etude $n^{\circ} 13$.

[4] BLOGOWSKI A., BOYER P., 1994 - Les effets différenciés de la réforme de la PAC sur les revenus des agriculteurs. Economie Rurale, 220-221, 124- 130 .

[5] Météorologie Nationale, Butletins climatiques mensuels. Service Central d'Exploitation de la Météorologie, Division Climatologie.

[6] TIRA M. , VINCENT B. , 1995 - La Loi sur l'Eau : ses incidences sur le drainage agricole. Géomètre, 7, 29-31. 